\title{
An Investigation of Motion of a Crawling Robot with Supports with Controllable Friction
}

\author{
L. Yu. Vorochaeva, S. I. Savin, A. S. Yatsun
}

This paper deals with the gait of a robot crawling on a horizontal rough surface with dry friction. A distinctive feature of the device is the presence of two supporting elements with a controlled coefficient of friction, which allows for alternately fixing the supports on the surface. As a result of numerical simulation, the patterns (laws) of influence on the motion characteristics of the mass-dimensional and control parameters of the robot, as well as the parameters of the supporting surface, are obtained, so that it is possible to find application in the design of the specified devices.

Keywords: crawling robot, supporting element, controlled friction coefficient, dry friction model

\section{Introduction}

Currently, scientists from different countries are conducting research aimed at creating crawling robots to carry out rescue, search, and reconnaissance operations in completely or partially destroyed buildings. The choice of exactly these crawling devices as such robots is due to their higher maneuverability and terrain crossing capacity over a non-deterministic surface in the presence of obstacles, as well as the ability to penetrate through narrow restricted spaces (slots, pipes, ventilation shafts) that are most often observed after natural and man-inflicted disasters.

Received May 31, 2019

Accepted July 27, 2019

This work was carried out within the framework of the Presidential Grant MK-200.2019.1.

Ludmila Yu. Vorochaeva

mila180888@yandex.ru

Andrey S. Yatsun

ayatsun@yandex.ru

Southwest State University

ul. 50 let Oktryabrya 94, Kursk, 305040 Russia

Sergey I. Savin

s.savin@innopolis.ru

Innopolis University

ul. Universitetskaya 1, Innopolis, 420500 Russia

RUSSIAN JOURNAL OF NONLINEAR DYNAMICS, 2019, 15(4), 623-632 
In this connection, the articles [1-3] address issues related to the development of gaits of crawling robots, as well as issues of movement on surfaces with obstacles. For instance, in [1] the development of such an algorithm (of the gait) for the movement of a crawling snake-like robot on the surface with obstacles is described, so that the trajectory of movement of the robot is smooth. The study [2] presents a method for designing two gaits of a crawling robot moving through pipes and over a rough terrain, where the required configuration of the robot is formed by connecting curve segments. Spatial movement of the crawling snake-robot in the presence of obstacles is considered in [3], overcoming obstacles is provided by vertical and horizontal deformations of the robot's body in the presence of anisotropic friction between its links and the surface. The papers [4-6] present movement issues of the crawling robots in limited spaces, including pipes and along cables.

In this paper, we consider one of the simplest design schemes of a crawling robot, which consists of a link made in the form of a rod and supporting elements installed at its ends, which provides for switching between two values of the friction coefficient, one of which allows the support to be fixed on the surface, and the other, to slide over it. The aim of this work is to identify numerically the patterns of movement of the robot on a rough surface, which can later be used in the design of such devices.

\section{Description of the crawling robot}

In this paper we consider a crawling robot consisting of one link and two supporting elements with a controlled friction coefficient, moving along a horizontal rough surface in the plane Oxy (Fig. 1a).

The link of the robot is a rod with length $l$ and mass $m$. The center of mass of the link is located at the point of symmetry of the link, denoted as point $C$. Let the position of the point $O_{1}$ be set by the radius vector:

$$
r_{O 1}=\left(\begin{array}{ll}
x_{O 1} & y_{O 1}
\end{array}\right)^{T},
$$

where $x_{O 1}, y_{O 1}$ denote the coordinates of the point $O_{1}$ in the projections on the axis $O x$ and $O y$, and the angle of rotation of the link relative to the positive direction of the axis $O x$ is equal to $\varphi$. Then the position of the center of mass of the robot is determined by the formula

$$
r_{C}=\left(x_{O 1}+l \cos \varphi / 2 y_{O 1}+l \sin \varphi / 2\right)^{T} .
$$

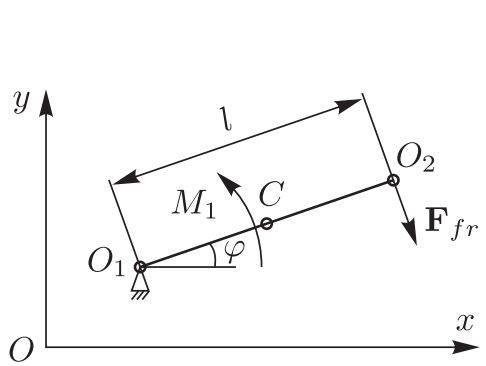

(a)

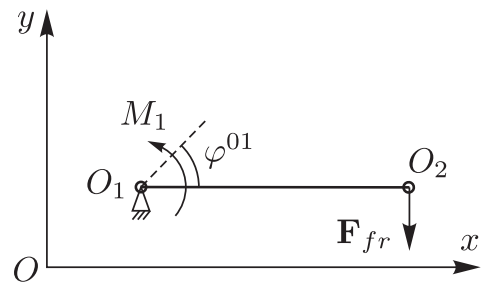

(b)

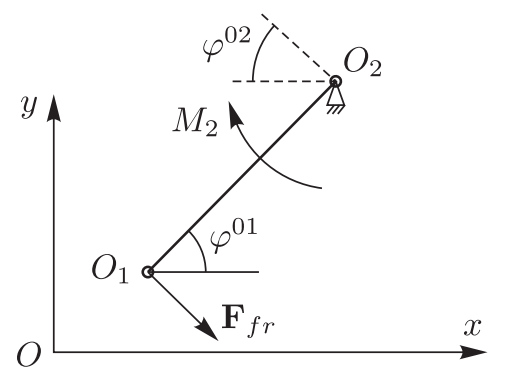

(c)

Fig. 1. a) A scheme of a crawling robot. b, c) Initial positions of the robot's motion stages: b) the first stage, c) the second stage. 
Supporting elements are set at points $O_{1}$ and $O_{2}$ and allow for switching between two possible values of the friction coefficient: maximal $f_{\max }$ and minimal $f_{\min }$ [7]. We assume that the value $f_{\max }$ is sufficient for the support to be fixed on the surface, while when the friction coefficient $f_{\min }$ is observed, the contact element slides along the surface. At the support points, the drives are installed which create the moments $M_{1}$ and $M_{2}$ and allow the robot link to rotate in a horizontal plane relative to the fixed support. In the second support, at the same moment, the dry friction force $F_{f r}$ arises.

\section{Friction force models}

In general terms, the dry friction force formula is written as

$$
F_{f r}=\left\{\begin{array}{l}
F_{d} \quad \text { if } \quad \dot{\varphi} \neq 0, \\
F_{s} \text { if } \quad(\dot{\varphi}=0) \wedge\left(\left|F_{0}\right| \leqslant F_{\text {pred }}\right), \\
F_{\text {per }} \quad \text { if } \quad(\dot{\varphi}=0) \wedge\left(\left|F_{0}\right|>F_{\text {pred }}\right),
\end{array}\right.
$$

where $F_{d}$ stands for the friction force in dynamics, $F_{s}$ denotes the static friction force, $F_{p e r}$ means the transient friction force, $F_{\text {pred }}$ is the ultimate friction force, $F_{0}$ is the resultant of all the forces except for the force of dry friction applied to the contact point of the robot, and $\dot{\varphi}$ denotes the angular velocity of the point of the robot in which the friction force is applied.

In this paper, we confine ourselves to three options for the force of dry friction: the force of symmetric dry friction (Fig. 2a), the friction force with a reduced dynamic component (Fig. 2b) and the friction force with a continuously drooping characteristic (Fig. 2c). Table 1 shows formulas for each of the friction forces.

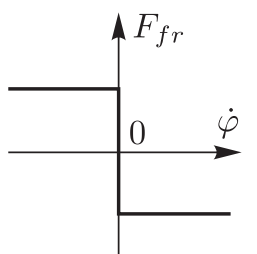

(a)

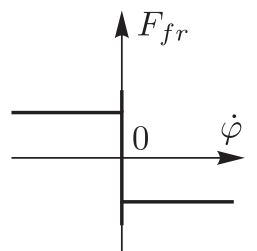

(b)

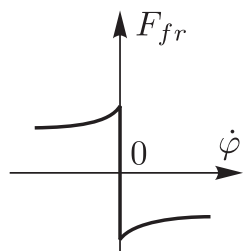

(c)

Fig. 2. Models of the dry friction force: a) symmetric, b) with a reduced dynamic component, c) with a continuously drooping characteristic.

Table 1. Formulas of models of the dry friction force

\begin{tabular}{|c|c|c|c|}
\hline $\begin{array}{c}\text { Friction } \\
\text { forces }\end{array}$ & Symmetric & $\begin{array}{c}\text { With a reduced dynamic } \\
\text { component }\end{array}$ & $\begin{array}{c}\text { With a continuously drooping } \\
\text { characteristic }\end{array}$ \\
\hline$F_{s}$ & \multicolumn{3}{|c|}{$-F_{0}$} \\
\hline$F_{\text {per }}$ & $-f_{\min } N \operatorname{sgn}\left(F_{0}\right)$ & $-f_{\min }^{s} N \operatorname{sgn}\left(F_{0}\right)$ & $-f_{\min } N \operatorname{sgn}\left(F_{0}\right)$ \\
\hline$F_{d}$ & $-f_{\min } N \operatorname{sgn}(\dot{\varphi})$ & $-f_{\min }^{d} N \operatorname{sgn}(\dot{\varphi})$ & $-f_{\min } N e^{-a|\dot{\varphi}|} \operatorname{sgn}(\dot{\varphi})$ \\
\hline$F_{\text {pred }}$ & $f_{\min } N$ & $f_{\min }^{s} N$ & $f_{\min } N$ \\
\hline$F_{0}$ & \multicolumn{3}{|c}{$M_{1,2} / l$} \\
\hline
\end{tabular}


Table 1 uses the following notation: $f_{\min }$ stands for the dry friction coefficient; $f_{\min }^{s}, f_{\min }^{d}$ denote the coefficients of the static friction and the slip, respectively, $N=m g / 2$ is the normal robot support reaction, and $a$ is the parameter determining the properties of a rough surface.

\section{Gait of the crawling robot}

By alternately controlling the friction coefficient in the contact elements, as well as intermittently feeding torques to the drives and turning them off, you can implement the gait of this robot, as shown in Figs. 1b, 1c. The robot's locomotion includes two stages. At the first stage $(n=1)$ the point $O_{1}$ is fixed. The drive set in this support generates torque $M_{1}$, which is formed until the equality $\varphi=\varphi^{01}$ is true, where $\varphi^{01}$ indicates some given value of the rotation angle of the robot link, corresponding to the completion of the first stage. After that, the second stage of the movement begins $(n=2)$, point $\mathrm{O}_{2}$ is fixed on the surface, the drive installed in it generates the torque $M_{2}$. The stage will be terminated when the condition $\varphi=-\varphi^{02}$ is satisfied, where the angle value $\varphi^{02}$ corresponds to the completion of the second stage. Then the stages are repeated. It should be noted that the values $\varphi^{01}$ and $\varphi^{02}$ are determined by the robot control system based on the trajectory along which the object must move, for example, when $\varphi^{01}=\varphi^{02}$ the movement will occur in a straight line, at $\varphi^{01}>\varphi^{02}$ it will be counterclockwise rotation, and at $\varphi^{01}<\varphi^{02}$, clockwise rotation. More explicitly the gait of the robot is illustrated in Table 2 .

Table 2. Description of the crawling robot gait

\begin{tabular}{|c|c|c|c|c|c|}
\hline & Motion $O_{1}$ & Motion $O_{2}$ & $f_{O 1}$ & $f_{O 2}$ & Termination condition \\
\hline stage 1 & - & + & $f_{\max }$ & $f_{\min }$ & $\varphi=\varphi^{01}$ \\
\hline stage 2 & + & - & $f_{\min }$ & $f_{\max }$ & $\varphi=-\varphi^{02}$ \\
\hline
\end{tabular}

The differential equation of motion of the robot during each of the stages is written as

$$
J \ddot{\varphi}=M_{1,2}-F_{f r} l,
$$

where $J$ stands for the moment of inertia relative to the fixed support point.

Let the moment of the drive in support 1 be changed according to the law

$$
M_{1}=\left\{\begin{array}{l}
M_{0} \sin \left(t-T_{1}^{*}\right) \quad \text { if }\left[M_{0} \sin \left(t-T_{1}^{*}\right)<M_{c}\right] \wedge[n=1], \\
M_{c} \text { if }\left[\left(M_{0} \sin \left(t-T_{1}^{*}\right) \geqslant M_{c}\right) \vee\left(M_{1}=M_{c}\right)\right] \wedge[n=1], \\
0 \quad \text { if }[n=2],
\end{array}\right.
$$

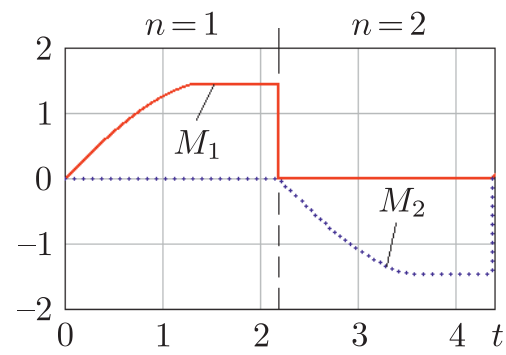

Fig. 3. Dependence graphs of $M_{1}(t), M_{2}(t)$. 
where $M_{0}$ denotes the moment amplitude, $T_{1}^{*}$ is the moment of time at which the equality $\varphi=\varphi^{01}$ holds, $M_{c}$ stands for the constant drive torque for which the condition $M_{c}<M_{0}$ holds true.

The moment of the drive in support 2 is described by the formula

$$
M_{2}=\left\{\begin{array}{l}
0 \quad \text { if } \quad[n=1], \\
-M_{0} \sin \left(t-T_{2}^{*}\right) \quad \text { if }\left[-M_{0} \sin \left(t-T_{2}^{*}\right)>-M_{c}\right] \wedge[n=2], \\
-M_{c} \quad \text { if }\left[\left(-M_{0} \sin \left(t-T_{2}^{*}\right) \leqslant-M_{c}\right) \vee\left(M_{2}=-M_{c}\right)\right] \wedge[n=2],
\end{array}\right.
$$

where $T_{2}^{*}$ denotes the moment of time at which the equality $\varphi=\varphi^{02}$ holds. The drive torque graphs are shown in Fig. 3.

\section{Simulation results}

Let us consider the rectilinear motion of the robot with the introduced models of friction forces with dimensionless values of the parameters: $m=1, l=1, M_{0}=1.5, M_{c}=1.45$, $f_{\min }=f_{\min }^{s}=0.2, f_{\min }^{d}=0.1, a=0.4, \varphi^{01}=\varphi^{02}=\varphi_{0}=0.5$.

Simulation of the robot's motion allows for the identification of the influence on the characteristics of movement of the mass-dimensional (the length and the mass of the link) and control parameters (the value of the control moments, the laws of their changes, the angle of rotation of the link), as well as the parameters of the supporting surface (the value of the friction coefficient, the model of the friction force). Figure 4 shows the time dependence of the change in the coordinates of the extreme points of the robot and its center of mass in the projections on the axes $O x$ and $O y$, as well as the trajectory graphs constructed for the symmetric dry friction model.

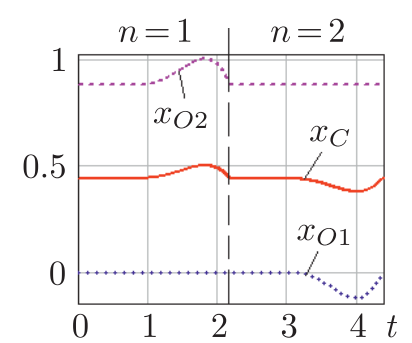

(a)

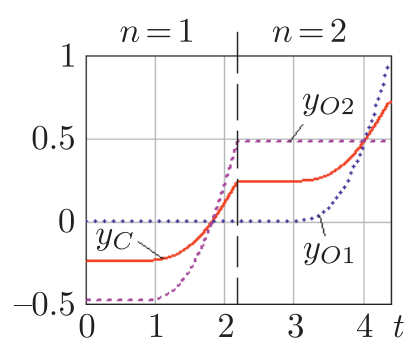

(b)

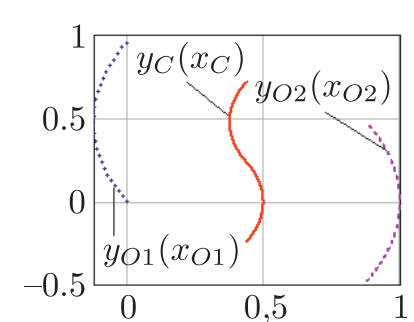

(c)

Fig. 4. Dependence graphs: a) $x_{O 1}(t), x_{O 2}(t), x_{C}(t)$, b) $y_{O 1}(t), y_{O 2}(t), y_{C}(t)$, c) $y_{O 1}\left(x_{O 1}\right), y_{O 2}\left(x_{O 2}\right)$, $y_{C}\left(x_{C}\right)$.

According to the graphs, it can be seen that during the first stage of the motion the points $O_{2}$ and $C$ dislocate, and during the second the points $O_{1}$ and $C$ dislocate, moreover, the movement occurs along the arcs of circles, the radius of which for the extreme points of the robot is twice larger than the radius for its center of mass. During each of the stages there are areas corresponding to the stationary state of the robot. This is due to the laws of the moment variation in the drives used in the work and the model of friction force (dry friction). It should be noted that the qualitative character of the graphs of Fig. 4 will not change with the variation of the model of dry friction force, and the quantitative changes will be due to the values of the static friction coefficients $f_{\min }^{s}$ and the slip $f_{\min }^{d}$ for the friction force with a reduced 
dynamic component and the coefficient value $a$ for the friction force with a constantly drooping characteristic.

In Fig. 5 the graphs of time dependences of the friction forces are shown during one cycle of movement (of two stages), where the areas of the static state of the object are highlighted in color. At these time intervals, the graphs of the friction forces repeat the corresponding laws of change of the control moments generated by the drives. In dynamics, the values of friction forces are either constant (for the symmetric friction force and the friction force with a reduced dynamic component, and in the first case the value of the sliding friction force is equal to the largest value of the static friction force, and in the second case is $f_{s} / f_{d}$ times less) either changed exponentially (for the friction force with a constantly drooping characteristic).

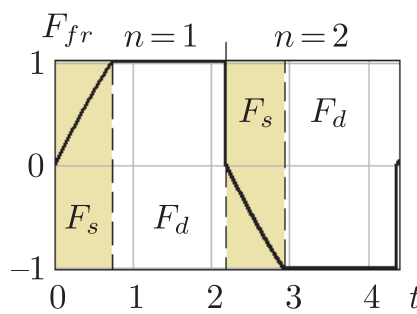

(a)

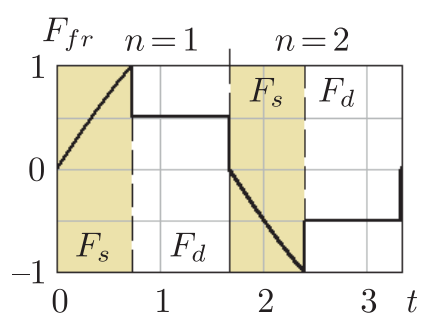

(b)

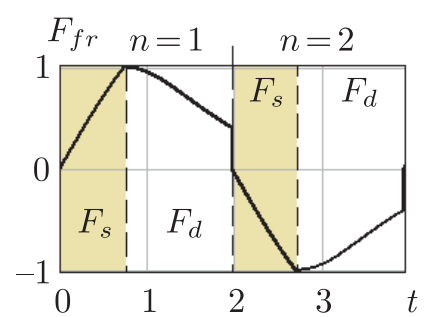

(c)

Fig. 5. Dependence graphs of $F_{f r}(t)$ in one cycle of motion with the models of friction forces: a) symmetric, b) with a reduced dynamic component, c) with a constantly drooping characteristic.

Based on these graphs, it can be concluded that the areas of the static position of the robot do not depend on the model of the friction force, but are determined by the value of the friction coefficient $f_{\min }=f_{\min }^{s}$, the larger this value, the wider the static area $t_{s}$ on the time interval of each stage $T_{1}^{*}=T_{2}^{*}=T^{*}$. In addition, the boundaries of this area depend on the weight-dimensional parameters of the robot (the mass $m$ and the length of the link $l$ ), and also on the moment $M_{c}$, generated by the drives, as illustrated in the diagrams of Fig. 6 .

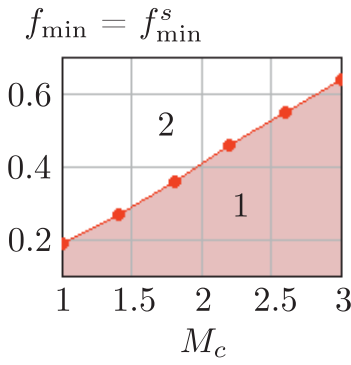

(a)

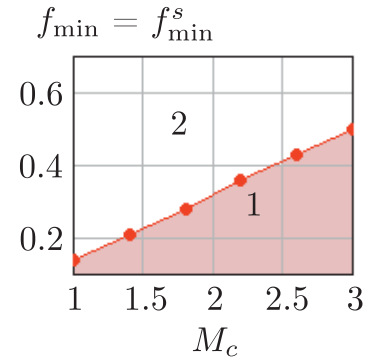

(b)

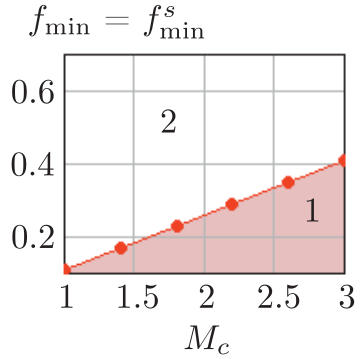

(c)

Fig. 6. Section (area) diagrams of $f_{\min }, f_{\min }^{s}\left(M_{c}\right)$ : a) $m, l=0.8$, b) $m, l=1$, c) $m, l=1.2,1$ - the range of values when the robot's movement is possible, 2 - the range of values at which the robot is in a stationary position.

Figure 6 shows the ranges of the coefficients $f_{\min }=f_{\min }^{s}$, at which the movement of the robot is possible (area 1) and in which the object is stationary (area 2, in it $t_{s}=T^{*}$ ) depending on the moment $M_{c}$ for three mass values $m$ of the link at $l=1$ or, on the contrary, for three lengths $l$ of the link at $m=1$. The given graphs indicate that, as the value $M_{c}$ increases, the maximum permissible value of the friction coefficient increases, at which the robot will move 
along the surface. Moreover, the dependence $f_{\min }, f_{\min }^{s}\left(M_{c}\right)$ is linear, the slope of a straight line to the axis of moments decreases as the mass or the length of the link increases.

Further simulation was carried out for such values of friction coefficients at which $t_{s}<T^{*}$. Its goal is to establish the patterns of influence of the parameters of the friction model on the time intervals $t_{s}$ and $T^{*}$. To assess the ratio of the time stage and the time during which the object is stationary, we introduce the ratio $t_{s}$ and $T^{*}$. To assess the ratio of the time stage and the time during which the object is stationary, we introduce the ratio

$$
p=\frac{t_{s}}{T^{*}} .
$$

The corresponding graphs are shown in Figs. 7-9. For the symmetric dry friction model, the friction coefficient $f_{\min }$ is considered as the parameter under study. According to the graphs in Fig. 7, it can be seen that the time of one stage of movement increases on the curve as the friction coefficient increases, and the greater the value of the drive torque $M_{c}$, the lower the graph of the corresponding curve is located.

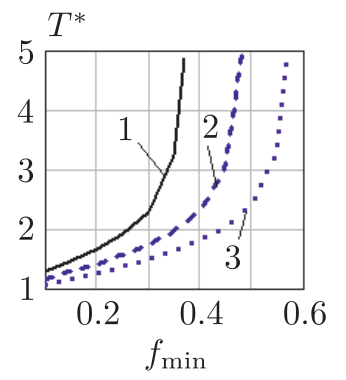

(a)

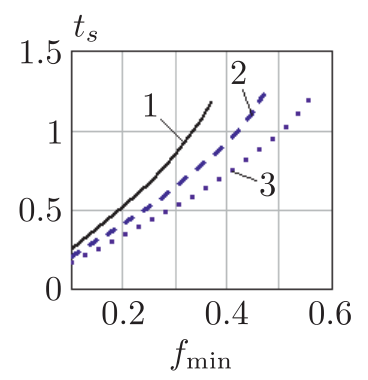

(b)

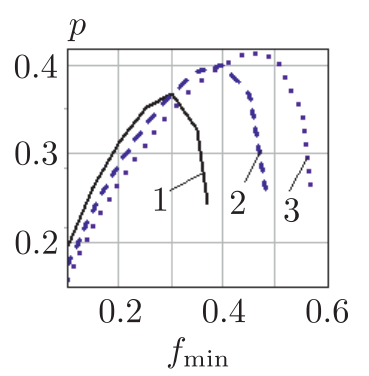

(c)

Fig. 7. Dependence graphs for symmetric dry friction force: a) $T^{*}\left(f_{\min }\right)$, b) $t_{s}\left(f_{\min }\right)$, c) $p\left(f_{\min }\right)$ at $1-$ $M_{c}=1.9,2-M_{c}=2.45,3-M_{c}=2.9$.

The time of the stationary state of the device in this case increases according to the laws close to proportional, the angle of inclination of the straight line corresponding to the largest value $M_{c}$, to the horizontal axis of the graph is the smallest (Fig. 7b). The ratio $p$ increases with the increase of the friction coefficient, reaches its highest value and then decreases, the nature of the curves is monotonic (Fig. $7 \mathrm{c}$ ). With the increasing $M_{c}$ value the highest parameter value $p$ is observed at a higher value $f_{\min }$ and this value itself increases in number.

For the other two models, the corresponding graphs are drawn at the moment of the drive $M_{c}=1.9$. For the model of friction force with a reduced dynamic component, the parameter under study is the coefficient of sliding friction $f_{\min }^{d}$. The time of each stage increases with the increase of $f_{\min }^{d}$ according to a curvilinear law, moreover, the greater the values of the coefficient of static friction $f_{\min }^{s}$, the higher is the corresponding curve (Fig. 8a). The time values $t_{s}$ do not depend on the coefficient $f_{\text {min }}^{d}$, and are determined only by the coefficient $f_{\text {min }}^{s}$, the greater the coefficient, the greater the rest time (Fig. 8b). The parameter $p$ decreases with the increase of $f_{\min }^{d}$ on the curve, the convexity of which is directed upwards, in this case there is no peak value observed (Fig. 8c).

The graphs in Fig. 10 for the friction force with a constantly drooping characteristic are built for the variable parameter $a$ at three different values of the friction coefficient $f_{\min }$. The type of the dependences $T^{*}(a)$ differs from that given above in Figs. 7 and 8, herein, there is a decrease in the time of the stage with the increase of the parameter $a$ on the curves, close to 


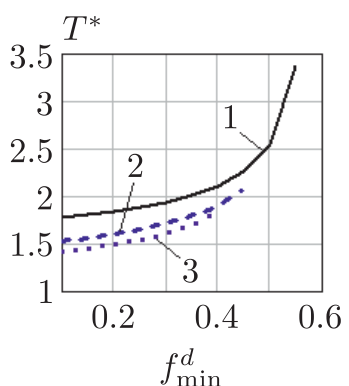

(a)

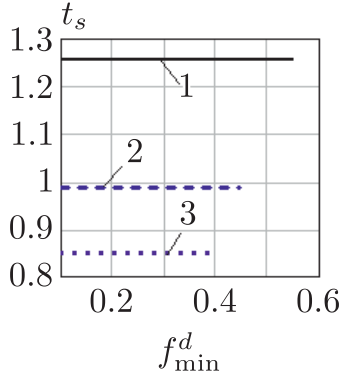

(b)

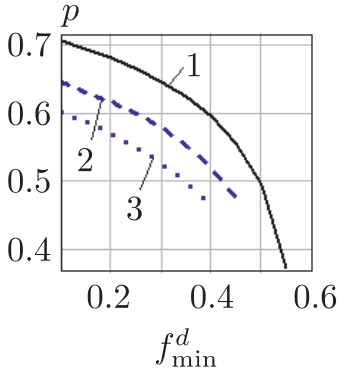

(c)

Fig. 8. Graphs of dependences for the friction force with a reduced dynamic component: a) $T^{*}\left(f_{\min }^{d}\right)$, b) $t_{s}\left(f_{\min }^{d}\right)$, c) $p\left(f_{\min }^{d}\right)$ at $1-f_{\min }^{s}=0.57,2-f_{\min }^{s}=0.5,3-f_{\min }^{s}=0.45$.

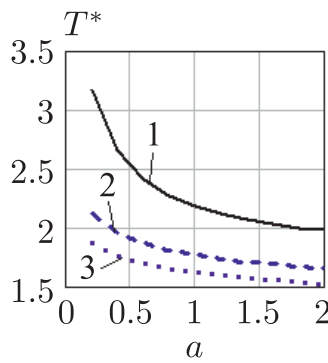

(a)

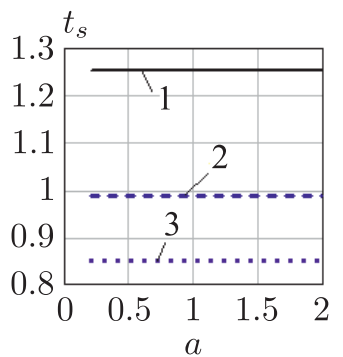

(b)

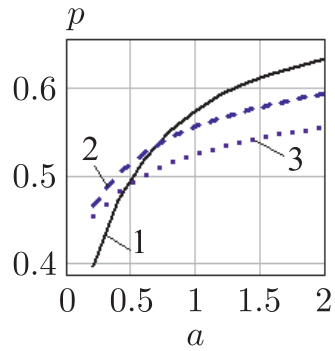

(c)

Fig. 9. Dependence graphs for the friction force with a constantly drooping characteristic: a) $T^{*}(a)$, b) $t_{s}(a)$, c) $p(a)$ at $1-f_{\min }=0.57,2-f_{\min }=0.5,3-f_{\min }=0.45$.

the exponentials, and with the increase of the friction coefficient $f_{\min }$ the corresponding curve is located higher relative to the horizontal axis of the graph (Fig. 9a).

The time of the object at rest (stationary state) (Fig. 9b), as well as for the force with a reduced dynamic component, does not depend on the variable parameter (in this case, the parameter $a$ ). The ratios of $p$ with the increasing parameter $a$ are increasing in the monotonic type according to curvilinear laws, the convexities of which are directed upwards; the ratio $p$ reaches the largest values at the highest of the considered parameter values of $a$ (Fig. 9c). Based on the graphs of Figs. 8-10, it can be concluded that for the most rapid movement of the robot on the surface it is necessary to ensure the minimum possible values of the friction coefficient $f_{\text {min }}$ for the symmetric friction force, the friction coefficients $f_{\min }^{s}$ and $f_{\min }^{d}$ for the friction force with a reduced dynamic component, the friction coefficient $f_{\min }$ and the parameter $a$ for the friction force with a continuously drooping characteristic.

Another variable parameter affecting the motion characteristics of the device is the value of the angle $\varphi_{0}$. To assess its impact, we have drawn graphs of the time of motion, $T$, it takes the robot to travel the distance $s=1$, in the event of variation of $\varphi_{0}$ with the three models of the friction force (Fig. 10).

The nature of these dependences does not depend on the model of the friction force; they are due only to the numerical characteristics of the curves. The graphs can be divided into three sections: in the first of them $\left(0 \leqslant \varphi_{0}<0.5\right)$ there is a decrease in time $T$ under the laws close to proportional, in the second $\left(0.5 \leqslant \varphi_{0}<1.4\right)$, the motion time does not depend on the angle $\varphi_{0}$, these areas feature horizontal lines, in the third $\left(1.4 \leqslant \varphi_{0} \leqslant 1.57\right)$, a decrease in the motion time is observed again as the angle $\varphi_{0}$ grows. 


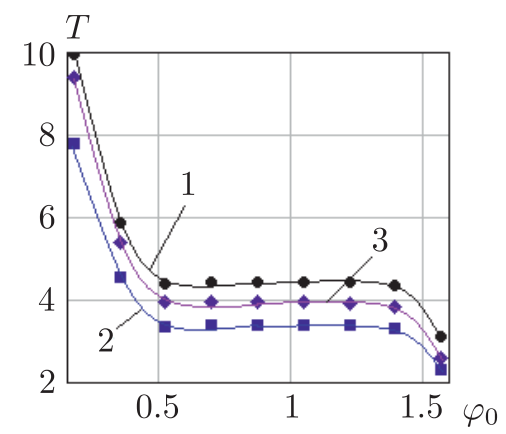

Fig. 10. Dependence graphs of $T\left(\varphi_{0}\right)$ with the friction models: 1 - symmetric, 2 - with a reduced dynamic component, 3 - with a constantly drooping characteristic.

\section{Conclusion}

This paper considers the movement of a crawling robot formed by a link in the form of a rod and two supporting elements with a controlled friction coefficient due to the change of the supporting surfaces. The gait of the device is proposed, consisting of alternate fixation of the supports and the rotation of the link relative to the fixed support. A simulation has been carried out of the movement of the robot on a horizontal rough surface, the interaction of which with the supports of the robot is described by three models of dry friction force: symmetric, with a reduced dynamic component, and with a constantly drooping characteristic.

As a result of this research, the influence on the motion characteristics of the massdimensional parameters of the device, control values and the parameters of the supporting surface has been established. It was revealed that, to increase the velocity of the robot when moving over the surface, it is necessary to minimize its mass $m$ and the length of the link $l$, as well as to use the largest possible moments $M_{c}$ of the drives and move at each stage to the largest possible angles $\varphi_{0}$. In addition, it has been established that the time of the robot's motion to a specified distance is reduced while minimizing the friction coefficients $f_{\min }, f_{\min }^{s}$ and $f_{\min }^{d}$ and the parameter $a$ for the appropriate friction models. The diagrams of the static and dynamic areas of the robot are drawn depending on the mass-dimensional and control parameters, it is established that the qualitative and quantitative characteristics of these diagrams are determined by the value of the static friction coefficient $f_{\min }$ or $f_{\min }^{s}$, but do not depend on the parameters of the model of the friction force, manifested in dynamics. The revealed regularities can be used in the development of the device design, the elaboration of the laws for controlling its drives, and in choosing materials for the supporting elements.

\section{References}

[1] Conkur, E.S. and Gurbuz, R., Path Planning Algorithm for Snake-Like Robots, Inform. Technol. Contr., 2008, vol. 37, no.2. pp. 159-162.

[2] Takemori, T., Tanaka, M., and Matsuno, F., Gait Design for a Snake Robot by Connecting Curve Segments and Experimental Demonstration, IEEE Trans. on Robotics, 2018, vol.34, no. 5, pp. 13841391.

[3] Fu, Q., Mitchel, Th., Yi, N., Gart, S., and Li, Ch., Snake Robot's Poor 3-D Obstacle Traversal Reveals Snake's Better Stability Mechanisms, APS March Meeting 2018, 2018, vol. 63, no. 1.

[4] Douadi, L., Spinello, D., Gueaieb, W., and Sarfraz, H., Planar Kinematics Analysis of a Snake-Like Robot, Robotica, 2014, vol. 32, no. 5, pp. 659-675.

RUSSIAN JOURNAL OF NONLINEAR DYNAMICS, 2019, 15(4), 623-632 
[5] Wei, W., Yu, J.X., Lin, W.H., Mai, R. Sh., Chen, Ch. X., He, Zh. Y., and Li, Zh., Structure Design of Climbing Snake-Like Robot for Detection of Cable-Stayed Bridge, Appl. Mech. Mater., 2014, vol. 598, pp. 610-618.

[6] Huang, Ch.-W., Huang, Ch.-H., Hung, Y.-H., and Chang, Ch.-Y., Sensing Pipes of a Nuclear Power Mechanism Using Low-Cost Snake Robot, Adv. Mech. Eng., 2018, vol. 10, no. 6, 8 pp.

[7] Vorochaeva, L. Yu., Yatsun, A. S., and Yatsun, S. F. Simulation of the Motion of a Five-Link Crawling Robot with Controlled Friction on a Surface Having Obstacles, J. Comput. Syst. Sci. Int., 2017, vol. 56, no. 3, pp. 527-552. 